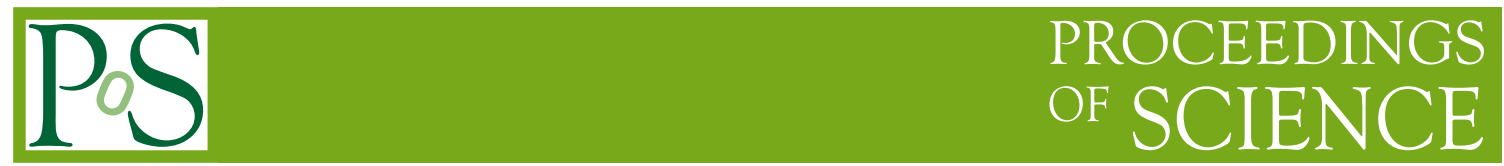

\title{
Beam Background Effects in the CMS Pixel Detector
}

\author{
Hella Snoek University of Zurich (On behalf of the CMS collaboration) \\ E-mail: hella.snoek@cern.ch
}

During the LHC run in December 2009 beam-induced background events were identified with the CMS pixel detector. The background consists mostly of collimated particle jets that traverse CMS parallel to the proton beam. The rate and origin of these events were studied, as well as the possible effects on the lifetime of the detector. In addition, a pixel-based method to remove the background for physics analyses was developed.

19th International Workshop on Vertex Detectors

June 6 - 112010

Loch Lomond, Scotland, UK

${ }^{*}$ Speaker. 


\section{Introduction}

During the first data taking with the presence of the LHC beam in December 2009, events were observed with high local occupancy in the CMS pixel detector. These events were present in both single beam and double beam runs and occurred in both single beam bunch crossings as in colliding bunch crossings. The beam background events were studied in more detail. The event rate was calculated and methods were developed to discriminate these events from collision events. The CMS pixel detector was one of the CMS detectors that was very well suited to study these events as it is in close proximity to the LHC beam pipe.

First, the CMS experiment is presented in Section 2, followed by a description of the characteristics of the beam background events in Section 3. Methods to remove the beam background events from the recorded data sets are presented in Section 4. Conclusions are summarized in Section 5.

\section{The CMS experiment}

The Compact Muon Solenoid (CMS) detector is a multipurpose experiment located at the CERN LHC and is described in detail elsewhere [1]. The CMS experiment uses a right-handed coordinate system with the origin located at the nominal interaction point (IP). The $x$ axis is pointing to the centre of the LHC ring, the $y$ axis is perpendicular to the LHC plane and points upwards. The azimuthal angle $\phi$ is measured in the $x y$ plane with $\phi=0$ along the positive $\mathrm{x}$ axis. The $z$ axis points in the direction along the direction of beam 1.

The CMS pixel detector will be described here in more detail as it has provided the data that will be described in these proceedings. It consists of three barrel layers and four fan-blade end-cap disks (two on each side) of n-in-n pixelized silicon sensors, see Fig 1, and comprises 66 million pixels with a total sensitive surface of about $1 \mathrm{~m}^{2}$. The barrel layers have an active length of $53 \mathrm{~cm}$ and are located at average radii of 4.3, 7.3 and $10.2 \mathrm{~cm}$. The layers are numbered from the inner (1) to the outer layer (3). The end-cap disks instrument the regions between radii 4.8 and $14.4 \mathrm{~cm}$ at a mean longitudinal distances of 35.5 and $48.5 \mathrm{~cm}$ from the nominal interaction point.

The pixels have a dimension of $100 \mu \mathrm{m} \times 150 \mu \mathrm{m}$, with the larger pitch in the $z$ direction in the barrel and along $\phi$ in the disks. The sensor thickness is $285 \mu \mathrm{m}$ in the barrel and $270 \mu \mathrm{m}$ in the disks. The pixel detector is situated within the $3.8 \mathrm{~T}$ magnetic field of the solenoid.

Two elements of CMS were used to trigger the detector readout during the early data taking of CMS. First, the Beam Scintillator Counters (BSCs) [1] which are a series of scintillator tiles designed to provide hit and coincidence rates. They have a sensitivity in the pseudorapidity range of $3.23<|\eta|<4.65$ and are located at $\pm 10.86 \mathrm{~m}$ from the nominal interaction point. Second, the Beam Pick-up Timing for the eXperiments (BPTX) devices [1] which is located around the beam pipe at a distance of $\pm 175 \mathrm{~m}$ from the IP on either side and is designed to provide information on the bunch structure and timing. Each BSC is a set of 16 scintillator tiles which have a time resolution of $2 \mathrm{~ns}$. In the early data-taking conditions, a single BPTX hit or BSC hit will trigger the readout of the detector.

\section{Beam background events}

Although many types of LHC induced beam backgrounds can exist, we will focus on one type 


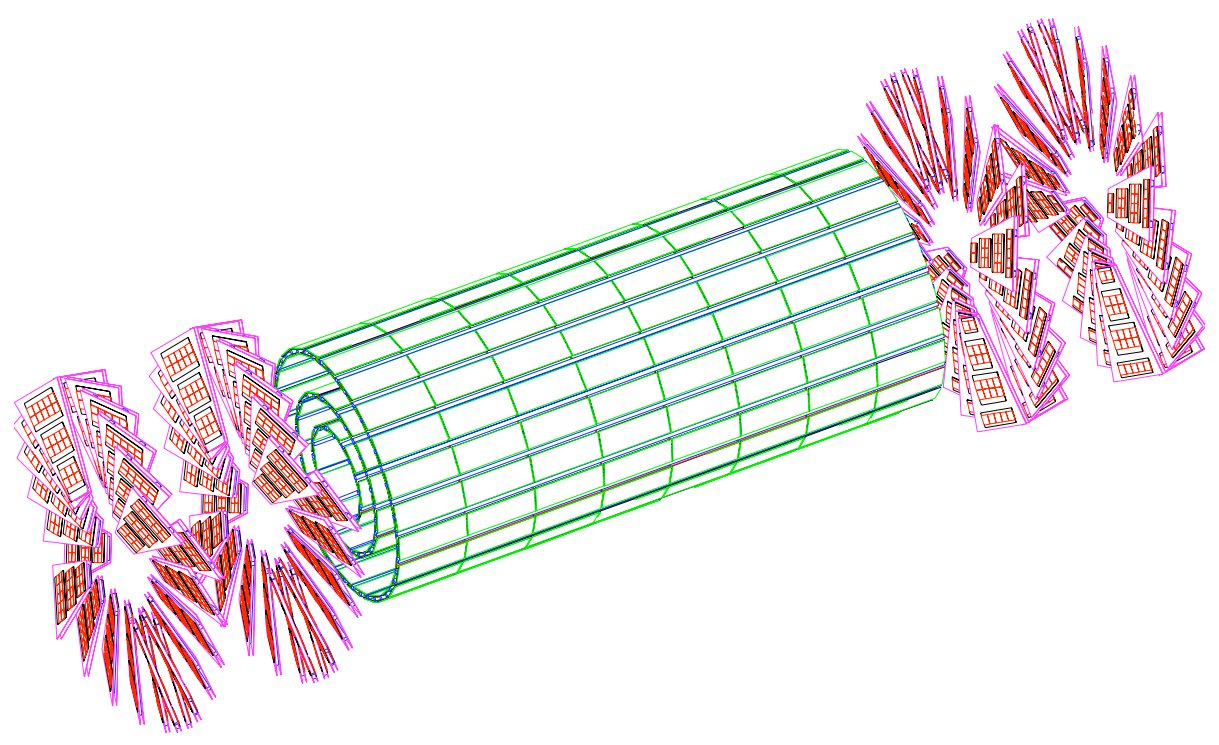

Figure 1: The CMS pixel detector

of beam background events and simply refer to these events as beam background events. These are the beam background events that are responsible for the high pixel-multiplicity events observed in the pixel detector, see Fig. 2. Several characteristics of these events have been studied that will be discussed here. From several studies that are described here it is found that the beam background events are compatible with beam interactions with residual gas in the beam pipe vacuum, that occur in proximity of the CMS detector (within a few hundred meters of the IP).

\subsection{Pixel multiplicity in the pixel detector}

Figure 2 presents the pixel multiplicity that was observed in the pixel detector at a $7 \mathrm{TeV}$ center-of-mass run. The distribution shows two exponentials. The exponential function with the high pixel multiplicity tail is associated to beam background events while the other exponential is associated to proton-proton collision events. The pixel multiplicity distribution in the pixel detector with the beam background events removed shows a good comparison with simulated data, see Ref. [3].

A comparison of the pixel multiplicity in beam background events for different beam energies (the single beam energy is half of the quoted center-of-mass energy) can be seen in Fig. 3. The plot clearly shows that the pixel multiplicity depends on the energy of the beam. The higher the beam energy, the higer the average pixel multiplicity in the pixel detector of the beam background events.

\subsection{Event topology}

A typical background event creates a lot of pixel hits in local regions of $\phi$ but along the full length in $z$ of the pixel detector. This is illustrated by Fig. 4 that show the location of the pixels that are above threshold in a single background event for the three different barrel layers. The background event is selected from a $7 \mathrm{TeV}$ run and has a 28,352 pixels over threshold. The location 


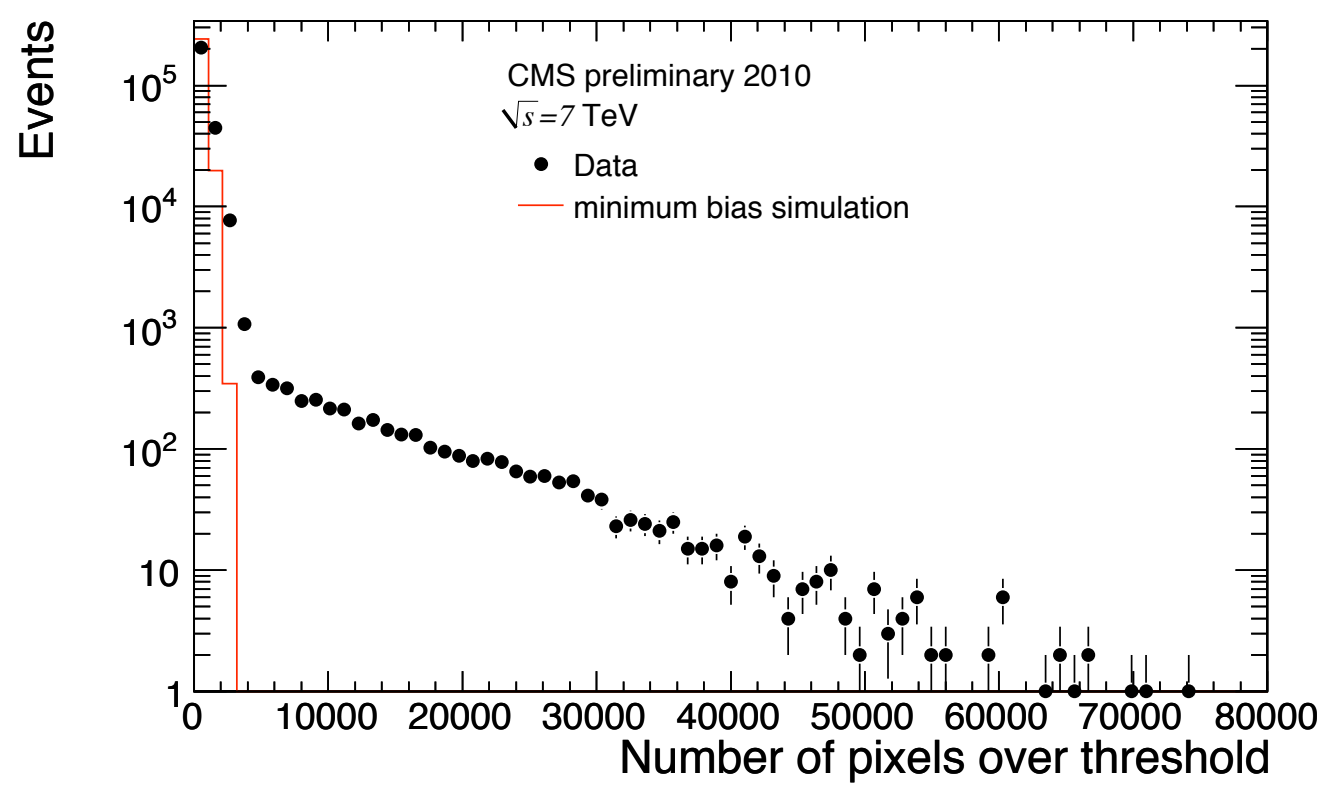

Figure 2: The pixel multiplicity in the pixel detector for the triggered events (black dots) overlaid to Monte Carlo simulation of minimum bias events (red line). In the observed pixel multiplicity distribution one can distinguish two exponentials. The events in the tail are associated to beam background events.

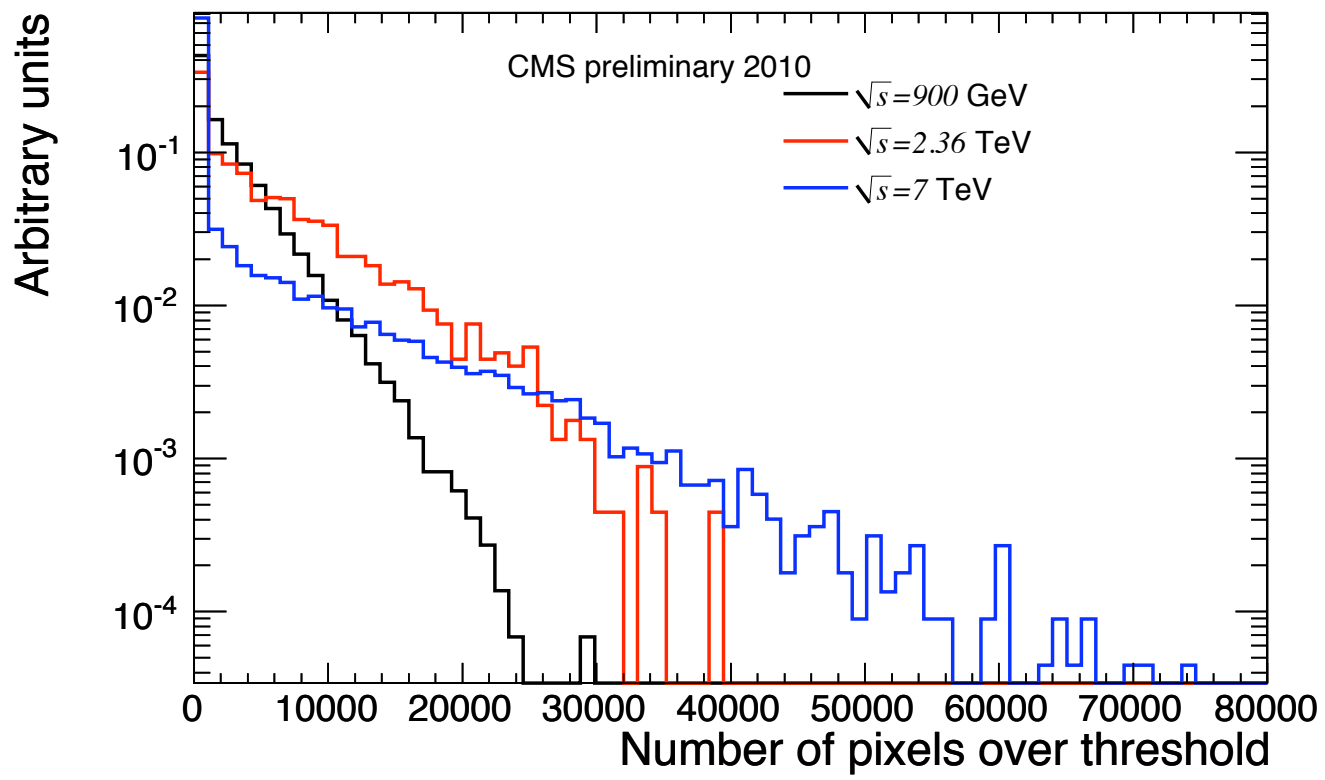

Figure 3: The plot shows the pixel multiplicity of the beam background events in the pixel detector for three different center of mass energy of the LHC beams. The events have been selected using the filter discussed in Section 4 and requiring more than 10 pixels over threshold in the event. 
of the pixels over threshold in the pixel disks, for the same background events, are shown in Fig. 5. This event coincides with the passing of beam 2 which goes in the $-\hat{z}$ direction. Here we observe that the charged particles, that create the hits in the pixel detector, enter the pixel volume from one side and leave on the other side. The particles enter and exit in the same region in $\phi$ and at the same radial distance from the beam pipe. From this typical topology for these beam background events we conclude that they consist of sprays of particles that fly along, and in close proximity of, the beam pipe.

Figure 6 shows a detailed view of two pixel readout modules of the innermost barrel layer of the same background event that is plotted in Fig. 4. We observe a cluster of over-threshold pixels that is almost $6 \mathrm{~cm}$ long in $\hat{z}$. This indicates that the charged particles that produce these clusters fly almost parallel to the $285 \mu m$-thick silicon.

The cluster that is visible around $\phi=-0.37$ and $-9 \lesssim z \lesssim 3$ is consistent with a charged particle decaying within the silicon volume. A particle that creates such a decay in the silicon is more likely to be hadronic than leptonic.

The particles that leave a long cluster in $\phi$ and narrow in $z$ are consistent with low momentum particles that are looping in the magnetic field.

Other clusters in the $\phi$ direction may originate from $\delta$ rays created in the silicon.

In the beam background events the local occupancy in the barrel detector can be very high, this is demonstrated in Fig. 4 and Fig. 6. This can cause problems in the data readout of the pixel detector. More information on this topic can be found in [8].

\subsection{Fake tracks}

The pixels over threshold are clusterized and transformed in so called reconstructed hits. The hits are then input to a tracking algorithm [5]. The background events leave many pixels over threshold in a single event and this creates many reconstructed hits. The event topology of the beam background events is such that the reconstructed hits are created within the same region of $\phi$ throughout all layers of the barrel and in the end-cap disks. This leads to many possible combinations of reconstructed hits from which tracks can be reconstructed, fullfilling all the requirement (e.g. pointing back to the IP region). These are fake tracks as they are not associated to a physical particle that can be associated with the same trajectory.

Figure 7 shows the number of reconstructed tracks per event. The observed distribution is overlaid to simulated minimum bias events. The distribution shows some similarities to the distribution of the number of pixels over threshold that is presented in Fig. 2. Again two exponentials can be distinguished. The tail with the high number of tracks is associated to the beam background events.

The presence of many fake tracks in these background events demonstrates the need to remove these events from physics analysis and detector performance studies. The removal of these type of events will be discussed in more detail in Section 4.

\subsection{Beam background event rate}

The beam background event rate is calculated and monitored by looking at single, non-colliding, bunch crossings and requiring BSC activity. The event rate has a linear dependence to the beam 


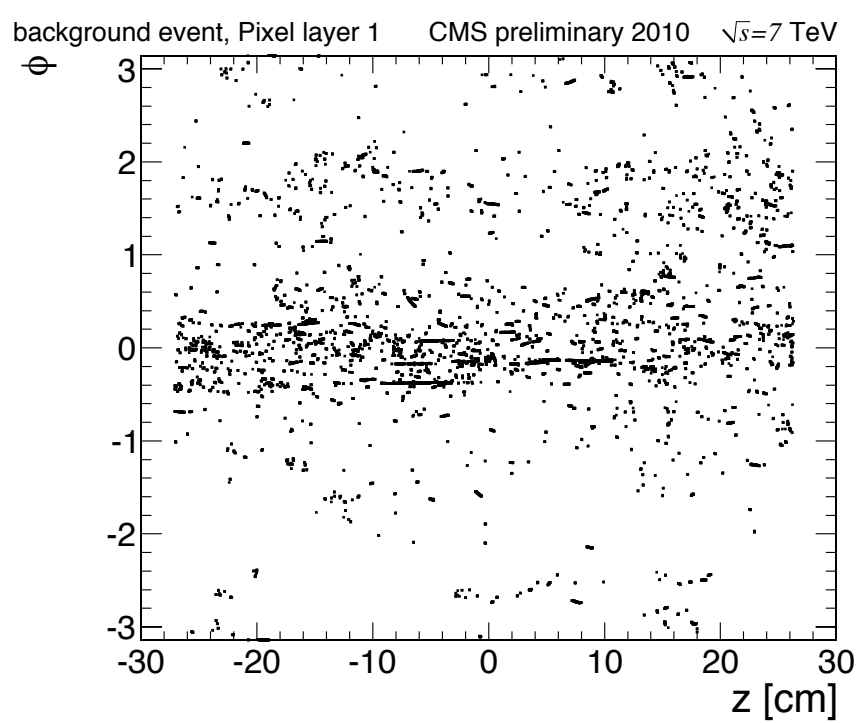

background event, Pixel layer $2 \quad$ CMS preliminary $2010 \quad \sqrt{s}=7 \mathrm{TeV}$

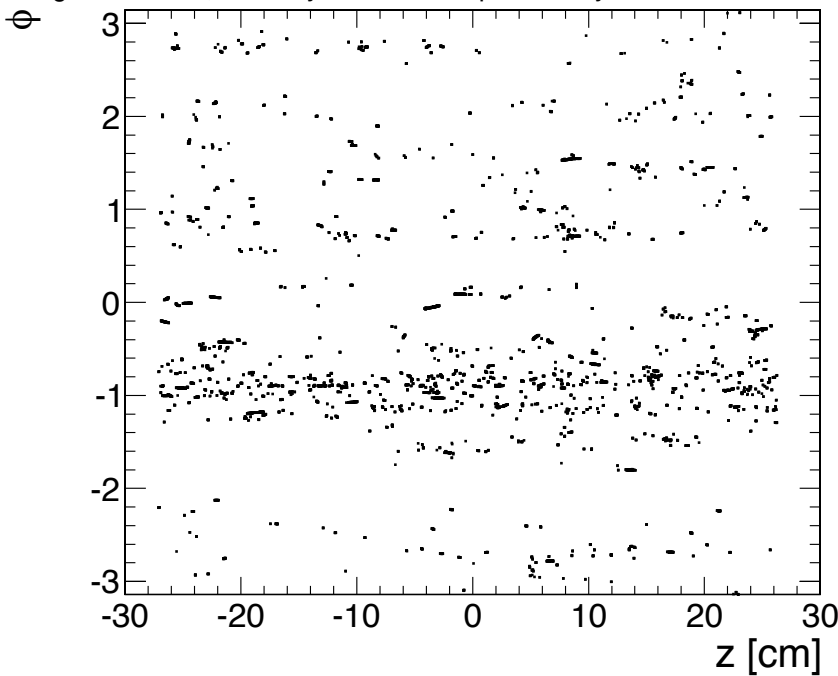

background event, Pixel layer $3 \quad$ CMS preliminary $2010 \quad \sqrt{s}=7$ TeV
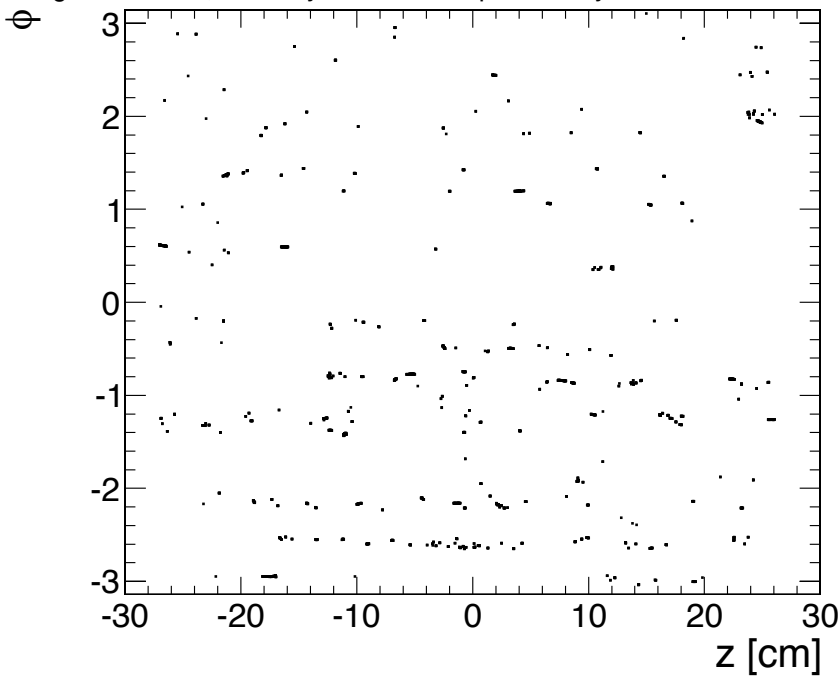

Figure 4: The location of the global $\phi$ versus $z$ location of all pixel hits (black rectangular) in a single background event per barrel layer. The layers are shown from the innermost at the top to the outermost at the bottom. 

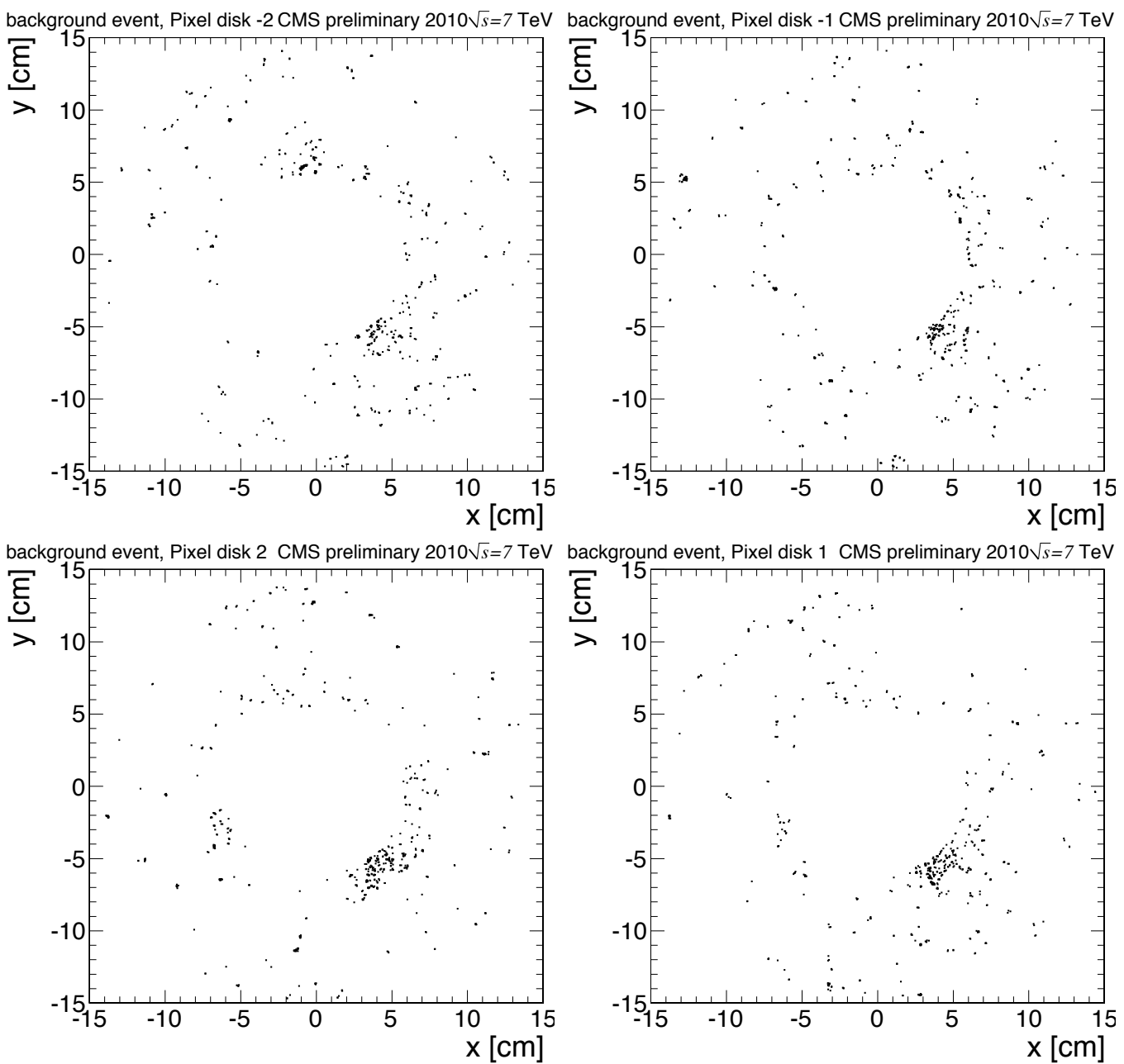

Figure 5: The global $x$ versus global $y$ location of all the pixel hits from a single background event for the four pixel disks. The top (bottom) two plots show the disks on the minus (positive) z side, the outer disks on the left and the inner disks on the right.

intensity. Fig. 8 shows the background event rate normalized to the beam intensity as function of time. The run lasted for 1.5 hours and during this time the beam background event rates were constant for both beams. Furthermore, the rate of the beam background events are the same for both beams, after normalizing to their beam intensities.

We have observed a constant normalized beam background event rate for all runs taken, independent of beam energies, beam intensities and beam parameters (such as $\beta^{*}$ ). The beam background rate is estimated to be approximately $0.5 \mathrm{~Hz} / 10^{10}$ protons/bunch/beam. This leads to the probability of a physics event overlapping with a beam background event of $1 \%$ for a given physics rate of $11 \mathrm{kHz}$ with $10^{11}$ protons per bunch. Even if one only triggers on collision events, the collected sample will be polluted with beam background events. This demonstrates again the need for an offline beam background rejection filter. 


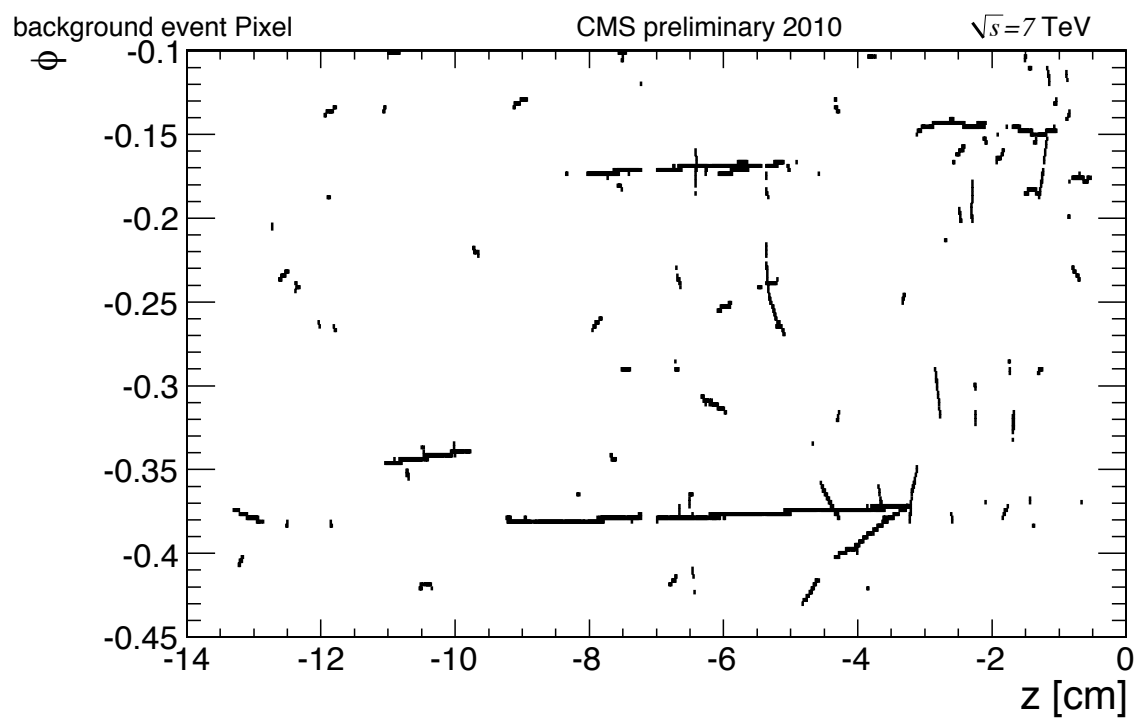

Figure 6: Pixels over threshold of two pixel modules in the innermost layer (1) of the barrel detector for a single background event.

\subsection{The beam-gas hypothesis}

There are different potential sources of beam background events and each would give their different characteristics of events. As already mentioned before the beam background events that are discussed in this paper are compatible with the hypothesis of beam-gas interactions.

All characteristics that have been described so far are in agreement with this hypothesis. A few more characteristics are summarized here.

- The observed 'spray of particles' (see Section 3.2) are in coincidence with the proton beam passing. This have been studied by looking at the timing information of the BSCs, the Hadron calorimeter (Forward) (HF) [1], and by looking at the cluster charge distributions in the pixels disks.

- The beam background is present in both colliding and non-colliding bunch crossings.

- The beam background is present in both beam passings.

Attempts have been made to reconstruct possible 'point-sources' around the IP that could be the origin of the spray of particles. For example protons from the beam could hit collimators or the beam pipe at places of narrow aperture, or beam shielding around CMS. In these studies the hits in the pixel end-cap disks are used to produce tracks. Vertices are then reconstructed using these tracks. This is highly non-trivial as one tries to extrapolate the tracks to many meters away from the IP using the hit information of the $\sim 1$ meter long pixel detector. The studies were able to identify the parasitic collisions that were present in some runs at $+11.25 \mathrm{~m}$ from the IP, but did not find any other significant increase of reconstructed vertices.

Furthermore the resulting particle density fluence has been compared with FLUKA based beam-gas interaction simulations [6]. The simulations have been used by the LHC to study the loss of protons from the LHC beam. Figure 9 shows the resulting track-length densities in the 


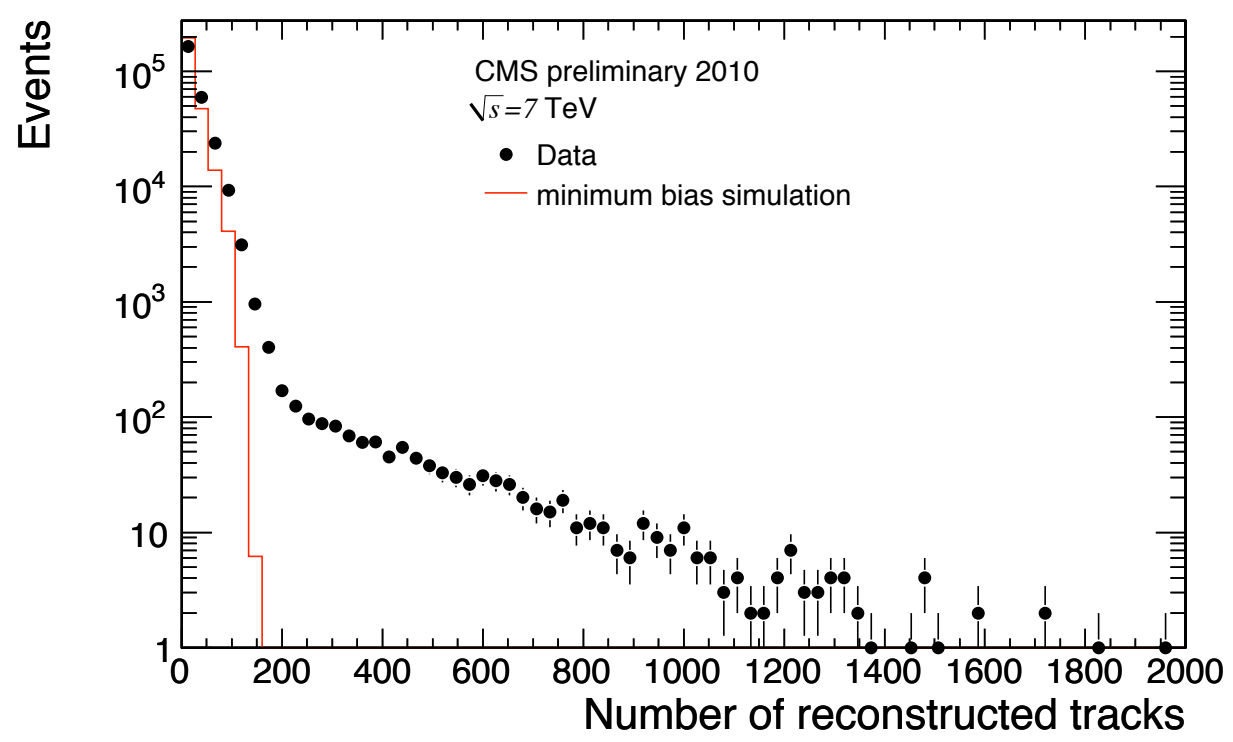

Figure 7: The plot shows the number of reconstructed tracks per triggered events (black dots) overlaid to Monte Carlo simulation of minimum bias events (red line). In the observed distribution one can distinguish two exponentials. The events in the tail are associated to beam background events.

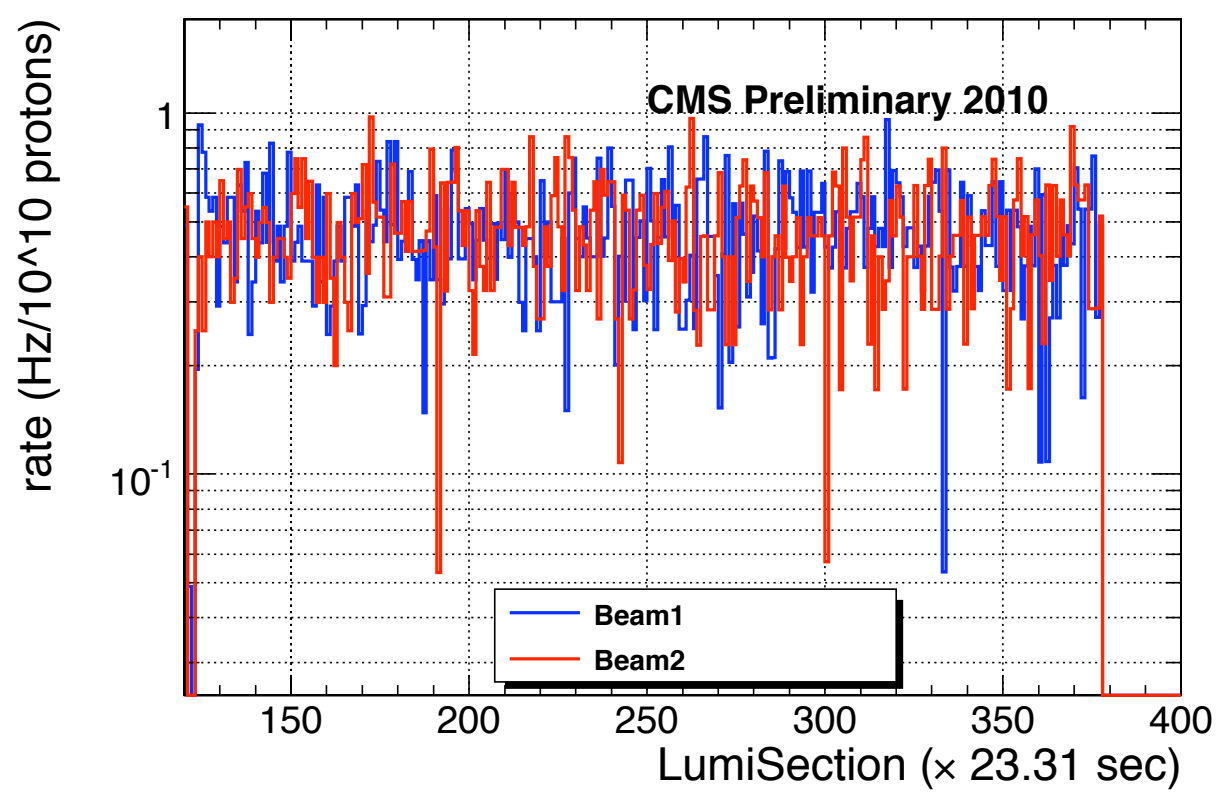

Figure 8: The beam background event rate versus the duration of the run. The rates are normalized to the beam intensity. Beam 1 (2) is shown in blue (red). The beam background events were selected by taking single (non-colliding) bunch crossings and requiring activity in the BSCs. The duration of the presented run is approximately 1.5 hours. 

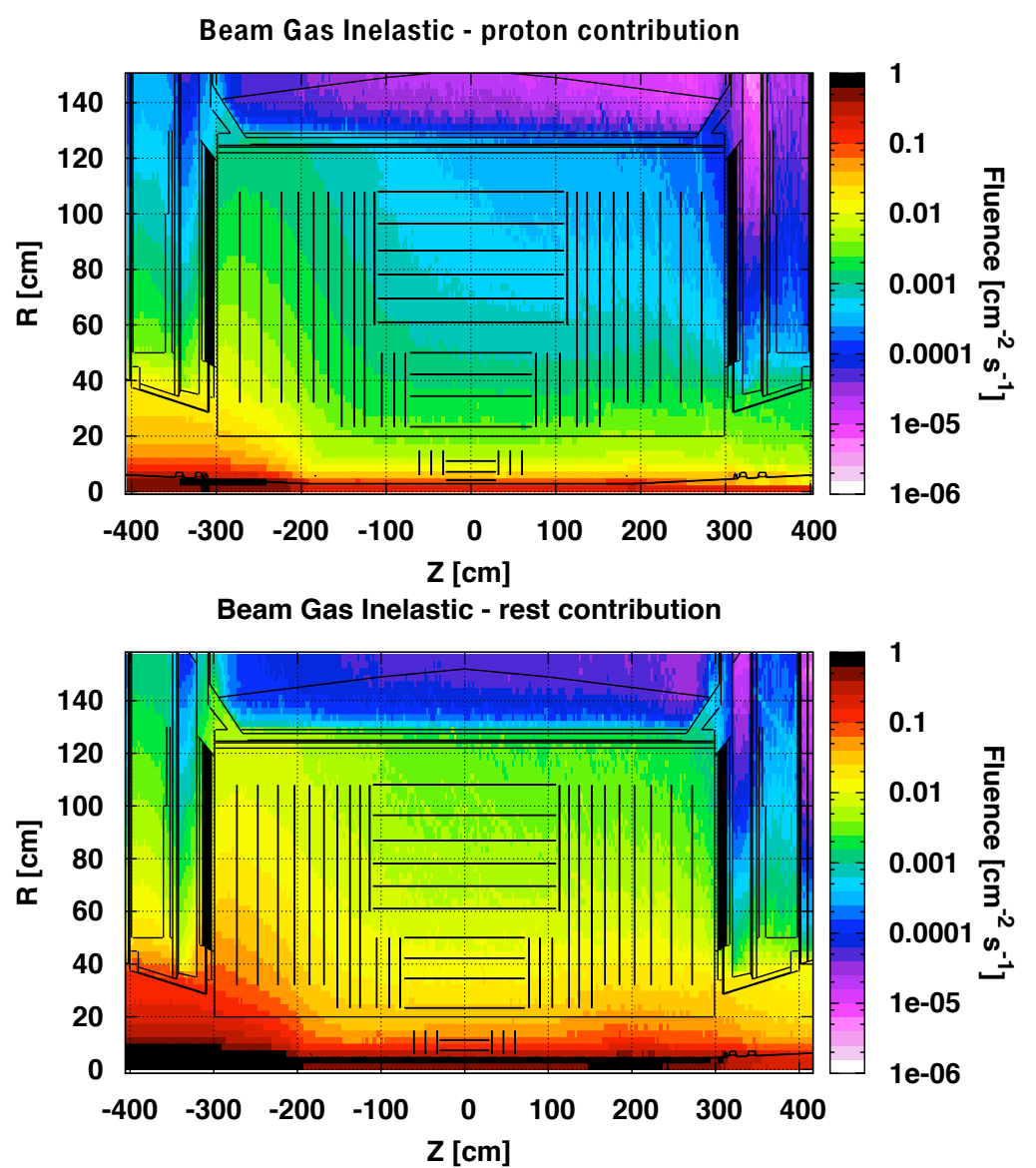

Figure 9: The resulting track-length densities per $10^{10}$ protons where inelastic beam 2-gas interactions have been simulated between 550 and 22 meters from the IP. Design vacuum conditions have been assumed. The upper plot shows the resulting proton contribution and the bottom plot shows the remaining contribution. This can be anything (p.e. photons) except for protons.

CMS detector area of simulated interactions between beam 2 (in the $-z$ direction) and residual gas between 550 and 2 meters from the IP. The simulated track-length densities are in agreement with the observed average of $\sim 50$ charged particles per event in the pixel disks and the normalized rate of $\sim 0.5 \mathrm{~Hz} / 10^{10}$ protons/bunch/beam.

\section{Rejection of beam background events}

The high number of fake tracks in a typical beam background event requires the removal of these events from the data sets. There are two ways of removing these events. First, to prevent triggering on these events and second is two remove them at the stage of the physics analysis.

To reduce the number of triggered beam background events can be easily done, p.e. by vetoing on single bunch crossings. The number of triggered background evetns can be easily reduced, p.e. by vetoing on single bunch crossings or using the timing information of the BSCs. However, to fully reject these events at triggering level will be very difficult. Also since there are events where a proton proton collision will overlap with a beam background event. 


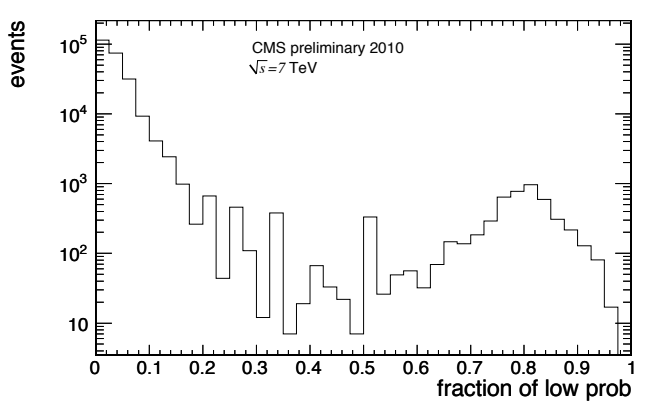

Figure 10: The fraction of clusters with a low probability per event for a $7 \mathrm{TeV}$ run.

This means that there is a need for offline filters to remove these events from the data samples that are used for physics analysis and performance studies. Different strategies can be used to discriminate the beam background events from the collision events. One can try to deploy the characteristics of the topology of the beam background events or p.e. use cuts on tracking quality. In the first published CMS physics analysis a beam background rejection was used that calculated the probability of the reconstructed hits in the event to be compatible with a primary vertex [2]. Other offline filters that are based on tracking, such as track purity and vertex qualities, have been used in the detector performance studies [3]. We describe here a filter that is based on the reconstructed hits in the pixel detector.

The filter makes use of the calculated template probability of the reconstructed pixel hit [7]. In the iterative tracking algorithm that CMS deploys the reconstructed hits, in the pixel detector, are refined using a template based method. The template fit uses the local impact angle of the track hypothesis and calculates the cluster matching probabilities (along the two local dimensions $x, y$, of the cluster and a comgined probability). Typically the hits on a fake track have a 'low probability' (defined as $\mathrm{P}_{x, y}\left(\chi^{2}\right)<9 \cdot 10^{-8}$, or $\mathrm{P}_{\text {comb }}\left(\chi^{2}\right)<2 \cdot 10^{-13}$ ). One can then calculate the fraction of low probability hits versus the total number of hits in an event. An event with a lot of fake tracks should than have a large fraction of low probability hits.

Figure 10 presents the calculated fraction of low probability clusters per event for a typical $7 \mathrm{TeV}$ run with the trigger conditions described in Section 2. Two regions are defined in the distribution with the fraction of low probability clusters higher or smaller than 0.4 . The region with the fraction smaller than 0.4 corresponds to the events with a low number of fake tracks which represent the proton-proton collisions. The region with the fraction higher than 0.4 corresponds to the events with a high number of fake tracks and these dominantly corresponds to the beam background events. Notice that the distribution is plotted in log scale and that the separation power is quite good. A template based filter can now be defined by selecting events with a fraction higher than 0.4 which we define, based on this filter, as beam background events or smaller than 0.4 which we define as collision events.

In Fig. 11 shows the pixel multiplicity and number of reconstructed tracks distributions where we have deployed the template based filter to separate the collision events from the beam background events. In both plots we notice that the two exponential tails are separated using the template based filter. The long tail is, as expected, associated to the beam gas events. 

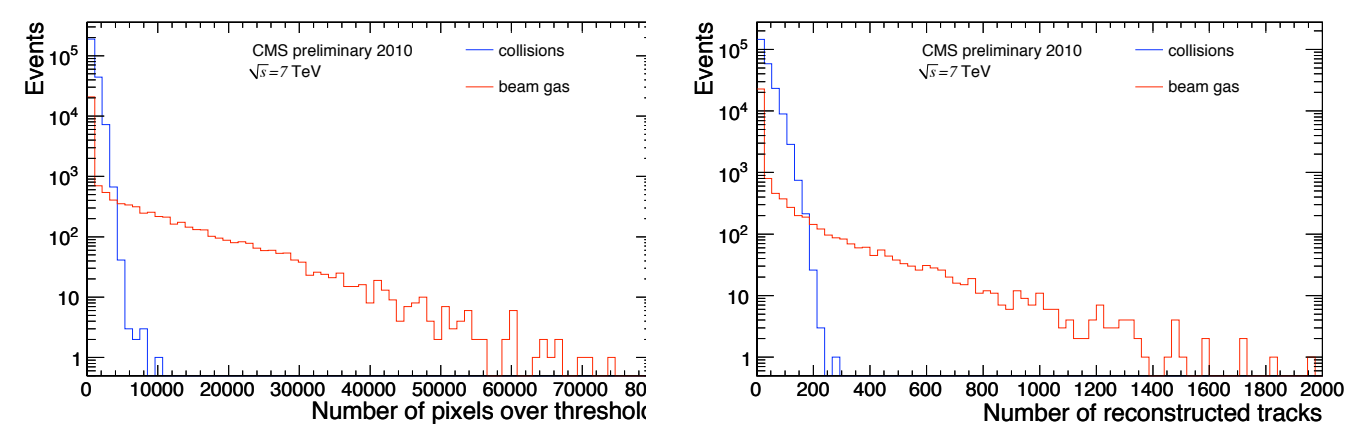

Figure 11: The left plot shows the pixel multiplicity in the pixel detector and the right plot shows the reconstructed-track multiplicity per event. In both plots the template-based filter has been used to separate the collision events from the beam background events.

\section{Conclusions}

We have observed events during first data taking with the presence of the LHC with a high pixel multiplicity in the pixel detector. The topology and frequency of the background events are consistent with beam-gas interactions. The beam intensity normalized rate of the events is 0.5 $\mathrm{Hz} / 10^{10}$ protons/bunch/beam. For a nominal physics run with $11 \mathrm{kHz}$ of pure physics events and and an intensity of $10^{11}$ protons per bunch this would result in $1 \%$ of the physics events overlapping with a background event. The fluence of the beam background events are consistent with the expectations from performed FLUKA simulations. Methods have been developed to effectively remove the beam background events from data analysis.

\section{References}

[1] R. Adolphi et al, (CMS Collaboration) The CMS experiment at the CERN LHC, JINST, 3 (2008) S08004.

[2] V. Khachatryan et al., (CMS Collaboration) Transverse momentum and pseudorapidity distributions of charged hadrons in pp collisions at sqrt( $s)=0.9$ and $2.36 \mathrm{TeV}, \mathrm{JHEP} \mathbf{0 2}$ (2010) 041 [hep-ex/1002.0621].

[3] V. Khachatryan et al., (CMS Collaboration) CMS Tracking Performance Results from Early LHC Operation [hep-ex/1007.1988v2].

[4] CMS Collaboration, Tracking and Primary Vertex Results in First 7 TeV Collisions CMS Physics Analysis Summary [TRK-10-005].

[5] W. Adam et al., Track Reconstruction in the CMS Tracker, CMS Note (2006) 2006-04.

[6] A. Ferrari et al., FLUKA: A multi-particle transport code (Program version 2005), CERN (2005) [CERN-2005-010].

[7] M. Swartz et al., A new technique for the reconstruction, validation, and simulation of hits in the CMS Pixel Detector, CMS Note (2007) 2007-033.

[8] J.M. Thompson, Operational Experience with the CMS Pixel Detector, in proceedings of 12th Topical Seminar on Innovative Particle and Radiation Detectors, submitted to Nuclear Physics B (Proceedings Supplement). 